\title{
Influence of polydispersity on the phase behavior of colloidal goethite
}

\author{
E. van den Pol, ${ }^{1, a)}$ D. M. E. Thies-Weesie, ${ }^{1}$ A. V. Petukhov, ${ }^{1}$ G. J. Vroege, ${ }^{1}$ and \\ K. Kvashnina ${ }^{2}$ \\ ${ }^{1}$ Van't Hoff Laboratory for Physical and Colloid Chemistry, Debye Institute for Nanomaterials Science, \\ Utrecht University, Padualaan 8, 3584 CH Utrecht, The Netherlands \\ ${ }^{2}$ DUBBLE beamline BM26, ESRF, 6 rue Jules Horowitz, BP 220, F-38043 Grenoble Cedex 9, France
}

(Received 20 June 2008; accepted 19 September 2008; published online 31 October 2008)

\begin{abstract}
The effect of fractionation on the phase behavior of colloidal goethite dispersions with different polydispersities $(17 \%, 35 \%$, and 55\% in length) has been studied by small angle x-ray scattering and transmission electron microscopy. All systems show at least nematic and smectic phases. The occurrence of the latter phase at such a high polydispersity is remarkable. It is shown that in the highly polydisperse systems strong fractionation occurs, which is able to reduce the local length polydispersity up to a factor of 2. A columnar phase was only found in the 35\% and 55\% polydisperse systems. It seems that the columnar phase accommodates the particles that do not fit into the smectic layers and, thus, reduces the length polydispersity within the smectic phase even further. The fact that a columnar phase was not found in the system of lowest polydispersity indicates that the smectic phase is the most stable phase at higher concentrations. () 2008 American Institute of Physics. [DOI: 10.1063/1.2999405]
\end{abstract}

\section{INTRODUCTION}

Lyotropic mineral liquid crystals were found for the first time by Zocher in $1925 .{ }^{1}$ Although considerably more attention has been paid to organic liquid crystals, which are well known for their applications like liquid crystal display screens, there is now renewed interest in mineral liquid crystals. ${ }^{2,3}$ Compared to organic liquid crystals, they have enhanced electrical, optical, and magnetic properties, together with a better thermal stability. ${ }^{4}$

An interesting example are colloidal dispersions of the mineral goethite $(\alpha-\mathrm{FeOOH})$. Special magneto-optical effects of aqueous colloidal suspensions of mixed iron oxides were already recognized by Majorana in 1902 (Ref. 5) and further studied by Cotton and Mouton in 1907. ${ }^{6}$ It turned out that one of the components was goethite. Goethite can easily form liquid crystals that are susceptible to external fields. Lemaire et $a l .{ }^{7-11}$ further revealed that the boardlike goethite particles have a permanent magnetic moment along their long axis, presumably due to uncompensated spins within their antiferromagnetic crystal structure, combined with an induced magnetic moment oriented predominantly along the shortest particle dimension. The interplay between them leads to peculiar field-strength-dependent reorientation phenomena.

In contrast to highly monodisperse viruses, ${ }^{12-15}$ colloidal dispersions of synthetic particles always have an inherent polydispersity. This polydispersity can sometimes suppress the formation of ordered phases. ${ }^{16,17}$ It was shown by computer simulation on hard spherocylinders, in the limit of infinite aspect ratio, that a smectic phase can only form if the length polydispersity is below $18 \% .{ }^{18}$ Above this terminal polydispersity, a columnar phase rather than a smectic phase

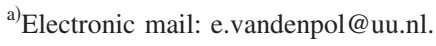

is expected at high volume fractions. A columnar phase can accommodate broader length distributions because particles do not have to fit into layers.

There are, however, recent theories that show that there is an influence of fractionation. It was first shown for bidisperse systems that fractionation occurs for isotropic-nematic phase separation in rod dispersions. ${ }^{19-21}$ The longer rods want to orient first and already form a nematic phase, while the shorter rods remain behind in the isotropic phase, which leads to a widened phase gap. Later, this was also demonstrated for continuous length distributions. ${ }^{22-25}$ Fractionation was also predicted for crystallization of polydisperse spheres. ${ }^{26}$ Different crystal phases can split off with a narrower size distribution than the parent phase. A terminal polydispersity still holds for each crystal phase, but the total system can have a higher polydispersity. The smectic and columnar phases have partial positional order and will probably fall in between the case of isotropic-nematic phase separation and crystallization, the smectic being particularly sensitive to the length distribution. In the context of small polydispersities the "universal law of fractionation" (of polydisperse particles partitioning over any two different coexisting phases) formulated by Evans et al. ${ }^{27}$ might be usefully applied.

Since colloids usually have a relatively high density, sedimentation is also an important factor in these systems. It was shown that the occurrence of multiple phases of monodisperse particles in a single test tube ${ }^{28}$ can partly be explained as a result of sedimentation. ${ }^{29}$ Sedimentation also couples to fractionation, as was recently shown for polydisperse van der Waals fluids. ${ }^{30}$

Recently, it was shown that goethite particles can form a smectic phase in a highly (over 50\%) polydisperse system. ${ }^{31}$ This was attributed to an interplay of sedimentation, fractionation, and Brownian motion. Furthermore, a columnar phase 
TABLE I. Goethite particle dimensions.

\begin{tabular}{lcccccc}
\hline \hline System & $\langle L\rangle(\mathrm{nm})$ & $\sigma_{L}(\%)$ & $\langle W\rangle(\mathrm{nm})$ & $\sigma_{W}(\%)$ & $\langle T\rangle(\mathrm{nm})$ & $\langle L\rangle /\langle W\rangle$ \\
\hline g55 & 216 & 55 & 35 & 48 & $\sim 16$ & 6.2 \\
g35 & 282 & 35 & 68 & 32 & $\sim 25$ & 4.1 \\
g17 & 220 & 17 & 62 & 29 & $\sim 23$ & 3.5 \\
\hline \hline
\end{tabular}

was found, together with the smectic phase, which might act as a "waste disposal" for ill-fitting particles. Because of new possibilities to make less polydisperse dispersions, ${ }^{32}$ it is now possible to study the effect of polydispersity on the phase behavior of goethite in greater detail. In this article the phase behavior of systems with different polydispersities is compared. A detailed measurement was performed by transmission electron microscopy (TEM), and in this way the real particle distributions at different heights in different phases could be determined.

\section{EXPERIMENTAL}

\section{A. Synthesis}

Different synthesis methods were used to obtain systems with different polydispersities, as was published earlier by Thies-Weesie et al. $^{32}$ The systems with a high polydispersity (g55 and g35) were obtained by hydrolysis of iron nitrate at high $p \mathrm{H}$ according to Lemaire et $a .^{8} 1 M \mathrm{NaOH}$ (Acros, reagent ACS, pellets, 97+\%) was added dropwise, under stirring, to a $0.1 M$ iron nitrate (Fisher Scientific, p.a.) solution until a $p \mathrm{H}$ of $11-12$ was reached. The precipitate was aged for 9 days after which the supernatant was removed, and the sediment was washed two times with doubly distilled (dd) water and $3 \mathrm{M} \mathrm{HNO}_{3}$ (Merck, p.a., 65\%) to electrostatically charge the particles by proton adsorption. After centrifuging and redispersing in dd water for three times the particles were redispersed in dd water to obtain a stable dispersion in water at $p \mathrm{H} 3$. The charge density at $p \mathrm{H} 3$ is around $0.2 \mathrm{C} \mathrm{m}^{-2}$. To obtain a lower polydispersity (g35) more centrifugation steps were used.

The system with the lowest polydispersity (g17) was obtained by a slightly adjusted forced hydrolysis method described by Krehula et al. ${ }^{33} 25 \mathrm{ml}$ of $25 \%$ tetramethyl ammonium hydroxide (Aldrich, 25\% w/w in water) was added, under vigorous stirring, to a solution of $0.16 \mathrm{M}$ iron nitrate. The solution was aged for 12 days at $100{ }^{\circ} \mathrm{C}$. The obtained dispersion was centrifuged and stabilized as described above.

It is assumed that after many centrifugation steps, the salt concentration is determined by the $\mathrm{H}^{+}$and chargecompensating $\mathrm{NO}_{3}^{-}$concentrations. The Debye length is then $10 \mathrm{~nm}$ at most.

\section{B. Characterization}

Particle size distributions were determined by TEM using a Technai 10 and 12 (FEI company) electron microscope. The particles have a more or less rectangular boardlike shape with three different dimensions: length $L$, width $W$, and thickness $T$. The length and width of about 500 particles were measured with iTEM imaging software to determine the average length $\langle L\rangle$ and width $\langle W\rangle$ and their standard devia- tion $\delta_{L}$ and $\delta_{W}$. The length polydispersity is then defined as $\sigma_{L}=\delta_{L} /\langle L\rangle$. Also correlations between the length and width were measured by measuring the length and width of each particle together. The thickness was difficult to measure because hardly any particles lay on their side on the TEM grid. For each sample about 10-20 particle thicknesses were measured.

Particle size distributions were also determined as a function of the height in the capillary. To this end, capillaries were cut into small pieces after first freezing them in liquid nitrogen. The pieces were put in small vials, after which water was added. The particles were homogeneously dispersed by ultrasonication and the different fractions were analyzed by TEM.

\section{SAXS experiments}

Samples with different volume fractions were prepared in flat glass capillaries (Vitrocom RT3524) with internal dimensions of $0.2 \times 4.0 \times 10 \mathrm{~mm}^{3}$. The capillaries were closed with two-component epoxy glue (Bison Kombi rapide) and kept in a vertical position to allow the establishment of the sedimentation equilibrium profile. The samples of the g55 system were made in November 2003, the ones of the g35 in January 2004, and of g17 in December 2004. To study the liquid crystalline phase behavior as a function of polydispersity and time, small angle $\mathrm{x}$-ray scattering (SAXS) measurements were performed. These measurements were performed at the BM-26 DUBBLE beamline of the European Synchrotron Radiation Facility (ESRF) (Grenoble, France) in February 2004 and November 2006. In 2006 the recently developed microradian resolution setup ${ }^{34}$ was used.

To be able to calculate distances from the scattering patterns, calibration samples were measured. In 2004 a dry rat tail collagen was used as a calibration sample and in 2006 a wet rat tail collagen and a $4.2 \mu \mathrm{m}$ grid were used. The two calibration samples of 2006 gave nearly identical results and therefore the calibration of 2006 is taken as the most reliable. Comparing the same sample in 2004 and 2006, there is a difference of about 5\%-10\%. For a better comparison, the measurements of 2004 are scaled on the measurements of 2006 by using a scaling factor based on this average difference.

\section{RESULTS AND DISCUSSION}

The particle dimensions of the systems used are shown in Table I.

As was shown before, ${ }^{31,32}$ systems with a high polydispersity (g35 and g55) show rich phase behavior. Isotropic $(I)$, nematic $(N)$, smectic A $(S)$, and columnar $(C)$ phases were found together in samples at a range of (initial) volume frac- 


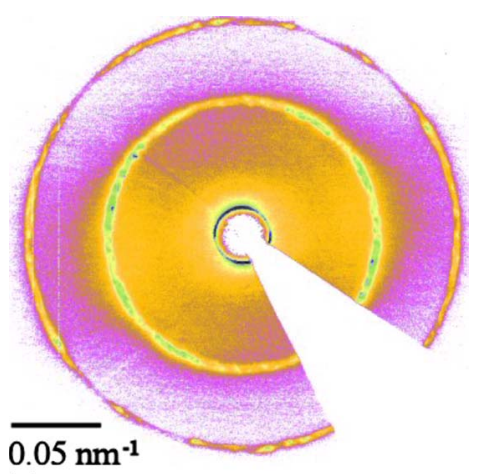

(a)

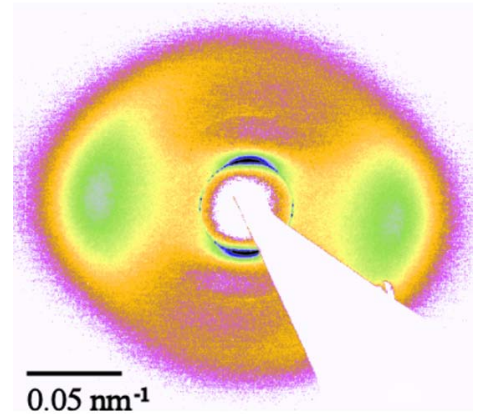

(b)

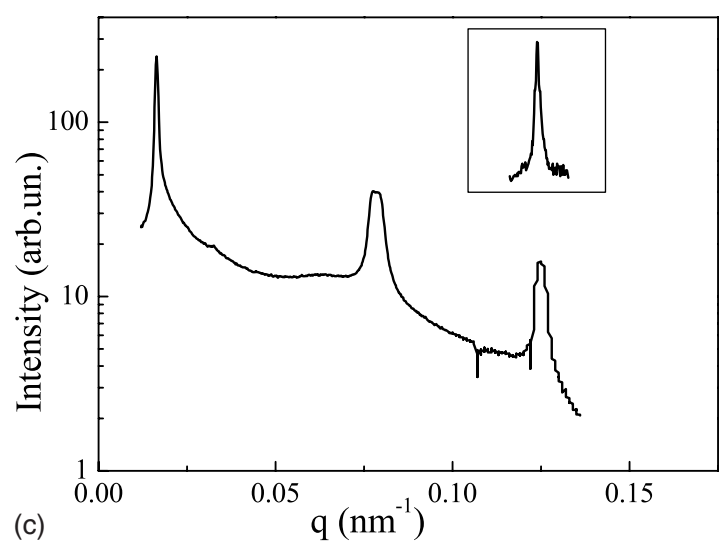

FIG. 1. (Color online) (a) SAXS pattern showing a coexisting smectic A and columnar phase (g35), (b) SAXS pattern of a single smectic A phase (g17), and (c) the integrated intensity profile of the pattern in (a) with a slice through one of the columnar peaks shown in the inset.

tions (5\%-10\% for $\mathrm{g} 35$ and $8 \%-18 \%$ for $\mathrm{g} 55)$. Here, we present a more detailed study of these systems.

In Fig. 1(a) an example is shown of the SAXS pattern of coexisting smectic and columnar phases which are observed low in the samples. The sharp scattering at small angle corresponds to the smectic periodicity, and the broad scattering peak, originating from the liquidlike interactions within the smectic layers, can be seen as a broad shoulder under the sharper columnar peak around $q=0.08 \mathrm{~nm}^{-1}$. The columnar phase is recognized by the powderlike sharp scattering rings. Because there are columnar domains with slightly different $q$ values, the integrated intensity profile shows broader peaks than expected for a columnar phase. In the inset of Fig. 1(c) a sharper peak is shown originating from only one of the domains. These sharp peaks originate from a distortedhexagonal intercolumnar structure $(\mathrm{c} 2 \mathrm{~mm}$ symmetry group $)^{10}$ and correspond here to distances of about 80 and
$50 \mathrm{~nm}$. This gives unit cell parameters of $100 \times 133 \mathrm{~nm}^{2}$ with two particles per unit cell. Comparing this with the average width $(68 \mathrm{~nm})$ and thickness $(25 \mathrm{~nm})$, it is clear that there is a lot of free space between the columns, although the fact that the columnar phase exhibits a distorted-hexagonal structure proves that the two smallest particle dimensions are not equivalent and that the particles are not able to rotate freely around their long axis in this columnar structure.

The occurrence of a smectic phase in highly polydisperse systems seems to be in contradiction with the terminal length polydispersity of $18 \%$ found for the smectic phase by simulations on hard spherocylinders. ${ }^{18}$ The boardlike goethite particles are compared with spherocylinder models because there is hardly any literature on particles with this exact shape. The difference in shape does not seem to be too important in the case of a smectic phase where the length polydispersity should be dominant. The smectic phase found was rationalized earlier by the interplay between sedimentation and fractionation. ${ }^{31}$ Furthermore, it is possible that particles that do not fit into the smectic phase are expelled and form a columnar phase, where particles with different lengths are allowed.

In the system with a low polydispersity (g17) only isotropic, nematic, and smectic A phases were found. The scattering pattern of the single smectic phase is shown in Fig. 1(b). No columnar phase was observed in any of the samples, at least up until a volume fraction of $14 \%$ and after standing for several years. In the highly polydisperse systems a columnar phase was already found at far lower volume fractions. This strengthens the idea of the columnar phase acting as a "waste disposal" for ill-fitting particles which is not needed for a system of low polydispersity. To gain more insight in this interesting phase behavior, a more detailed analysis was done.

\section{A. Fractionation}

The small angle smectic peaks originating from the periodicity of the layers give an indication of the length of the particles. This smectic periodicity is compared with the parent length distributions, measured by TEM, of the different systems, which can be seen in Fig. 2. The periodicity lies in the large distance tail of the length distribution for the systems with a high polydispersity. The ratio of the smectic periodicity from SAXS and the average length measured by TEM gives an indication of the extent of the fractionation. This ratio reduces from 1.85 for the g55 system to 1.40 for the g35 system. For the system of lowest polydispersity (g17), the smectic periodicity is close to the average particle size (ratio of 1.14). Thus, for a more polydisperse system, a stronger fractionation occurs.

Monte Carlo simulations of monodisperse hard spherocylinders have been done and the ratio between the periodicity of the smectic layers and the particle length that was found varied from 1.03 (Ref. 35) to $1.27 .{ }^{36} \mathrm{~A}$ problem in calculating this ratio is the occurrence of particles in between the smectic layers with an orientation which is more or less perpendicular to the particles in the layers. ${ }^{35,37}$ Experiments on Tobacco Mosaic Virus (TMV) found a factor of $1.12 .^{38}$ 


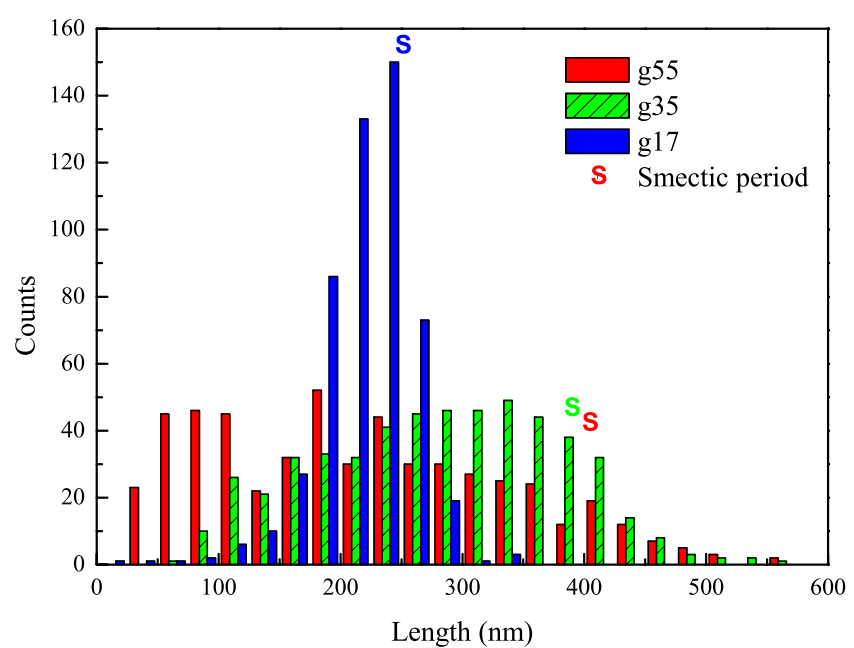

FIG. 2. (Color online) Length distributions of the parent suspensions of the g17, g35, and g55 systems. $S$ indicates the period of the smectic phase as measured in 2006 .

The TMV particles are charged as our particles. The ratio of the g17 system is close to that of the monodisperse TMV, suggesting that hardly any fractionation occurs.

\section{High polydispersity}

A more detailed analysis of the fractionation was done by TEM analysis of a capillary of the g55 system with an initial volume fraction of $8 \%$ instead of $16 \%$ which was used for the SAXS analysis. The averaged length and width, with their polydispersities, of fractions at different heights in the sample are shown in Table II. It can be seen that the average length drastically increases to the bottom of the capillary, indicating fractionation during sedimentation.

As indicated before, fractionation has already been predicted theoretically for isotropic-nematic phase separation in rod dispersions. ${ }^{19-25}$ We find that the polydispersity of the nematic phase is substantially lower compared to the isotropic phase. It was observed before that the polydispersity of coexisting isotropic and nematic phases in dispersions of natural clay rods is lower than that of the original dispersion. ${ }^{39}$ In our case, additional smectic and columnar phases form from the large particles of the nematic phase mainly, thereby further decreasing the polydispersity of the nematic phase.

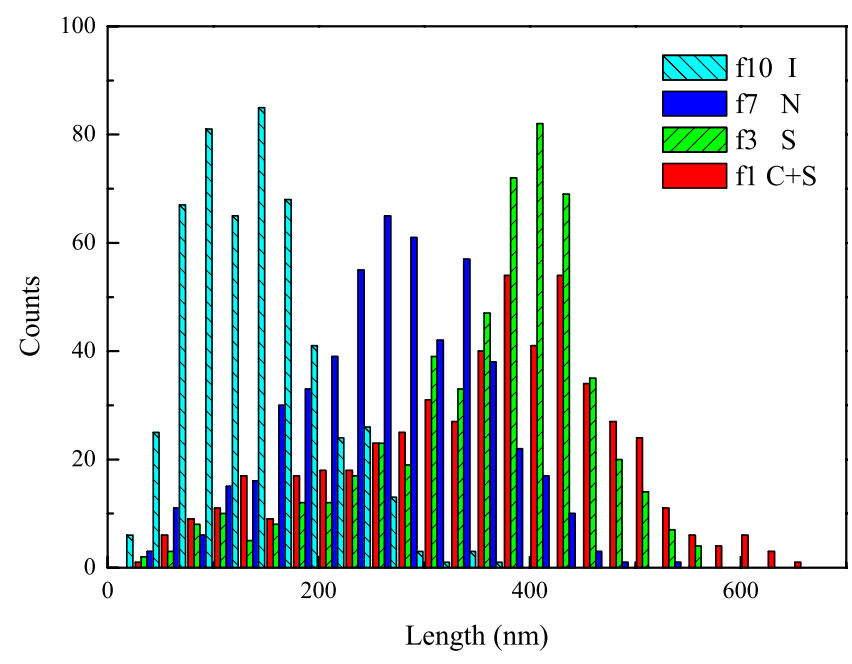

FIG. 3. (Color online) Length distributions of the fractions $f 1(C+S), f 3$ $(S), f 7(N)$, and $f 10(I)$ of the g55 system.

Interesting is the more or less constant particle length within the smectic region, while the polydispersity is lowest in the part where only a smectic phase occurs. The part where the smectic phase coexists with a columnar phase has a higher polydispersity, consistent with the notion that the columnar phase can accommodate particles that do not fit into the smectic layers. This can also be seen in the length distributions for some of the fractions, corresponding to different phases (Fig. 3). The distribution of the fraction of the pure smectic phase is the narrowest, although it still contains quite some shorter particles. It can be seen that the largest difference between the pure smectic phase and the coexisting smectic and columnar phases is in the presence of very long $(\sim 600 \mathrm{~nm})$ particles in the latter phase. Presumably, these largest particles cannot fit into the smectic layers but can be accommodated in the columnar phase.

The length polydispersity of $28 \%$ in the smectic phase still exceeds $18 \%$, as was found in simulations. ${ }^{18}$ There are some factors that might contribute to this. The breaking of the capillary is not very accurate; the breaking line was not always straight and precisely where it was supposed to be. In the $2.5 \mathrm{~mm}$ of the capillary which was used for the TEM analysis a change in the smectic periodicity of about $3 \%$ was found from SAXS. This of course increases the polydispersity found by TEM. Furthermore, the simulation was done

TABLE II. Averaged length and width of goethite particles as a function of height for the g55 system.

\begin{tabular}{rcccccc}
\hline \hline Fraction & Distance from the bottom $(\mathrm{mm})$ & Phase & $\langle L\rangle(\mathrm{nm})$ & $\sigma_{L}(\%)$ & $\langle W\rangle(\mathrm{nm})$ & $\sigma_{W}(\%)$ \\
\hline 10 & $41.5-55.0$ & $I$ & 132 & 47 & 28 & 47 \\
9 & $30.0-41.5$ & $I$ & 190 & 39 & 35 & 40 \\
8 & $21.5-30.0$ & $N$ & 228 & 33 & 40 & 39 \\
7 & $18.0-21.5$ & $N$ & 268 & 33 & 46 & 40 \\
6 & $15.5-18.0$ & $N$ & 304 & 30 & 52 & 35 \\
5 & $11.5-15.5$ & $N$ & 311 & 30 & 53 & 36 \\
4 & $9.0-11.5$ & $S+N$ & 332 & 30 & 54 & 41 \\
3 & $6.5-9.0$ & $S$ & 361 & 28 & 57 & 35 \\
2 & $3.5-6.5$ & $S+C$ & 364 & 33 & 54 & 54 \\
1 & $0-3.5$ & $S+C$ & 359 & 36 & 58 & 42 \\
\hline \hline
\end{tabular}


for hard spherocylinders with infinite aspect ratio, and these are soft-repulsive boardlike particles with an aspect ratio $(\langle L\rangle /\langle W\rangle)$ of 6.2. Finally, in the simulations the distribution of the length of the particles is Gaussian and thus symmetric. It is clear from Fig. 2 that the distribution of the g55 system is not symmetric, while the smectic distribution (Fig. 3) has a tail at small lengths, which would influence the simulation results.

For the average width the same trend was found as for the average length; the width increases going toward the bottom of the capillary. The lowest polydispersity is also found within the same range as for the length, although it is not expected to be important for the formation of a smectic phase. It seems that the length and width of the particles are strongly coupled-longer particles are also wider-although overall the fractionation in width is less pronounced than in length.

It is expected that the width polydispersity has a negative influence on the formation of a columnar phase, but this cannot be seen in these data. The columnar phase is always accompanied by a smectic phase, and therefore it is not possible to know the width polydispersity in the columnar phase only. Furthermore, the particles are not rodlike but boardlike which makes the situation more complicated. Finally, a relatively large influence of the double layer can contribute to the accommodation of a higher width polydispersity.

To determine the thickness, only about 10-20 particles could be measured so it is not possible to get good statistics out of it. A significant difference was only found for the two fractions in the isotropic phase: 8 and $13 \mathrm{~nm}$ compared to $20-24 \mathrm{~nm}$ for the other fractions. It is clear that there is a large difference in the volume of the particles going from the top to the bottom of the sample $\left(30 \times 10^{3}-500 \times 10^{3} \mathrm{~nm}^{3}\right)$. This corresponds to a gravitational length $\left(l_{g}\right)$ of $4.3-0.26 \mathrm{~mm}$, which is given by

$$
l_{g}=\frac{k_{B} T}{g \Delta \rho\langle L\rangle\langle W\rangle\langle T\rangle},
$$

where $k_{B} T$ is the thermal energy, $\Delta \rho$ is the mass density difference of goethite and water, and $g$ is the gravitational acceleration.

The aspect ratio $(\langle L\rangle /\langle W\rangle)$ increases from 4.7 to 6.7 going down in the capillary with a more or less constant value in the nematic phase. In Fig. 4(a) the individual aspect ratio $(L / W)$ is shown as a function of the length of the particles for some of the fractions. It can be seen that the aspect ratio of the particles increases with the length of the particles. Fractionation can be clearly observed; the different fractions have different average aspect ratios. Particles with a higher aspect ratio form more ordered phases. This is expected because they gain more free volume by aligning in ordered phases. Particles with a higher aspect ratio are also longer, so there is also fractionation in length.

A similar analysis of fractionation has been done for sepiolite clay. ${ }^{39}$ Fractionation in the length of the particles was found in the coexisting isotropic and nematic phases, but no fractionation in width was observed. There are some differences between the sepiolite and goethite systems that might explain this difference. Sepiolite is a natural clay as
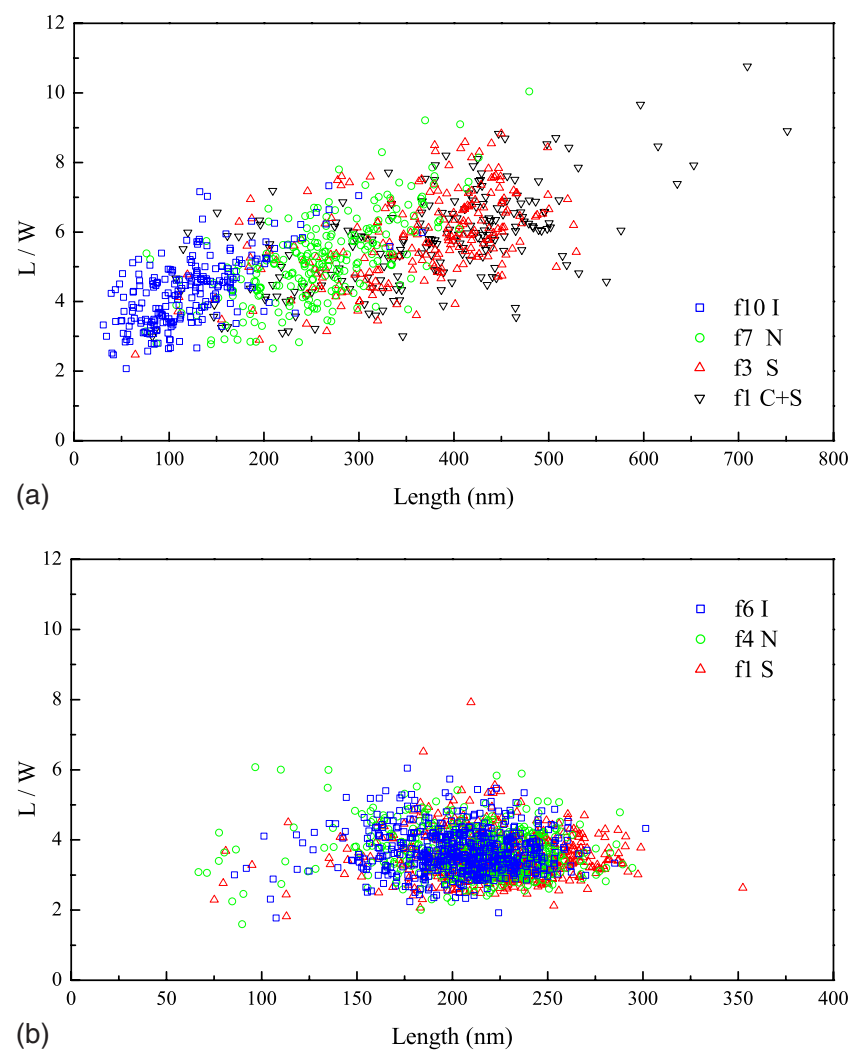

FIG. 4. (Color online) Aspect ratio $(L / W)$ as a function of length of fractions of the (a) g55 and (b) g17 systems.

opposed to the synthetic goethite. The lengths and widths of the sepiolite particles appear to be uncorrelated, which is not the case for the goethite particles [Fig. 4(a)]. Furthermore, the width polydispersity of the sepiolite particles is small $(15 \%)$ compared to that of the goethite particles studied here $(48 \%)$.

\section{Low polydispersity}

The same TEM analysis was done for a capillary of the g17 system. It can be seen from Table III that there is a slight increase in the particle length going down the capillary, but there is no difference in the polydispersity. Figure 5 shows that the length distributions of the lowest and highest fractions have the same shape; they are only slightly shifted. For the g55 system the differences in length and length distribution were much larger (see Fig. 3). This means that fractionation is much less pronounced for the goethite system of lowest polydispersity compared to highly polydisperse goethite.

It is important to point out that not only the width but also the symmetry of the parent length distribution plays a role here. ${ }^{27} \mathrm{~A}$ wider parent distribution gives rise to a larger difference between the average values of the daughter distributions, which is the case for our systems. Furthermore, a more asymmetrical distribution gives rise to a larger difference between the widths of the daughter distributions. The system with the lowest polydispersity is also the most symmetrical one, and the daughter distributions indeed have the same polydispersity, in accordance with the universal law of fractionation proposed by Evans et al. ${ }^{27}$ 
TABLE III. Averaged length of geothite particles as a function of height for the g17 system.

\begin{tabular}{ccccccc}
\hline \hline Fraction & Distance from the bottom $(\mathrm{mm})$ & Phase & $\langle L\rangle(\mathrm{nm})$ & $\sigma_{L}(\%)$ & $\langle W\rangle(\mathrm{nm})$ & $\sigma_{W}(\%)$ \\
\hline 6 & $20.5-24$ & $I$ & 204 & 15 & 57 & 22 \\
5 & $17.5-20.5$ & $I+N$ & 213 & 15 & 61 & 20 \\
4 & $13.5-17.5$ & $N$ & 212 & 17 & 60 & 25 \\
3 & $10.5-13.5$ & $S$ & 219 & 15 & 63 & 23 \\
2 & $7.5-10.5$ & $S$ & 221 & 15 & 66 & 22 \\
1 & $0-7.5$ & $S$ & 224 & 15 & 66 & 22 \\
\hline \hline
\end{tabular}

For the width the same trend was found as for the length: a little increase in the average width going down the sample and a more or less constant polydispersity. The aspect ratio $(\langle L / W\rangle)$ stays approximately the same over the whole sample. It can be seen in Fig. 4(b) that the aspect ratio $(L / W)$ is on average the same for all particles, so the small and large particles have the same shape. This is clearly different from the g55 system [Fig. 4(a)].

\section{B. Influence of time}

The changes in time give valuable information about the process of phase separation. In the first few months after preparation, already some changes could be seen. The lower initial volume fraction $(\sim 10 \%)$ capillaries showed isotropicnematic phase separation within a day, but it took several months to observe the development of smectic and columnar phases. This indicates that particles slowly sediment and fractionate and then phase separate. The capillaries with a higher initial volume fraction $(\sim 16 \%)$ showed smectic and columnar phases already after a week. In this case, it seems that fractionation and sedimentation-induced macroscopic separation, which are associated with the isotropic-nematic phase transition, help to create circumstances to form a smectic phase within a week. It is clear that for both processes Brownian motion is important, which is facilitated by the electric double layer around the particles.

One of the g55 samples, with an initial volume fraction of $16 \%$, has been measured by SAXS several times now. The first measurement of February 2004 (partly published in Ref.

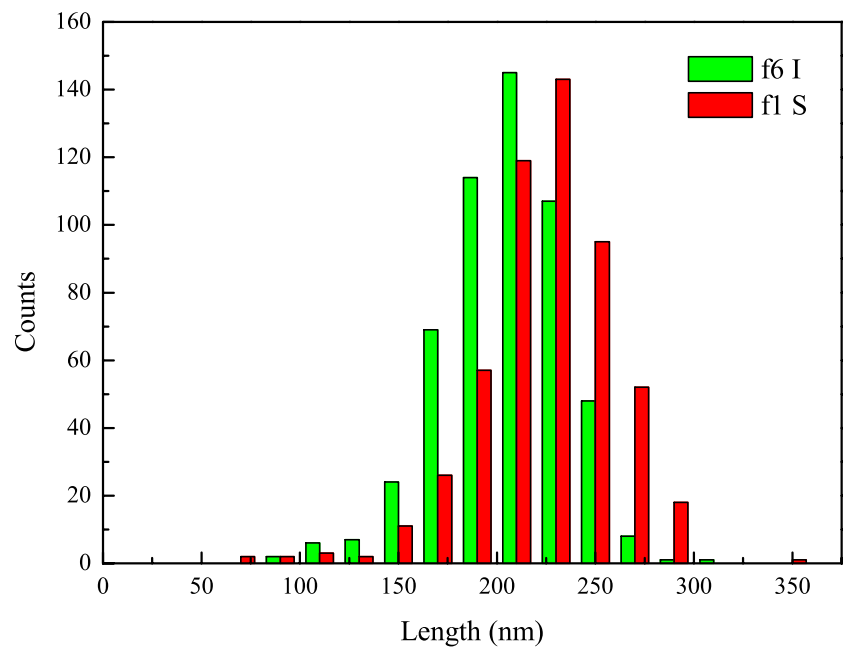

FIG. 5. (Color online) Length distributions of the fractions $f 1(S)$ and $f 6(I)$ of the g17 system.
31) is compared with the measurement of November 2006. The smectic and nematic distances at small angle, which give an indication of the particle length, are calculated from their $q$-values. The calibration of 2006 is taken as the most reliable one. Therefore, the measurements of 2004 are scaled on the ones of 2006, as described in Sec. II C.

It can be seen in Fig. 6 that in 2004, when the sample was 3 months old, there is a gradual increase in the distance going toward the bottom of the capillary. This is caused by a faster sedimentation of larger particles. In 2006, however, there are two regions with a more or less constant distance and a jump in the distance in between. This indicates a strong fractionation which makes it possible to form a much larger smectic phase. Down in the sample the smectic phase is accompanied by a columnar phase for the ill-fitting particles but higher in the sample even a pure smectic phase with a constant smectic periodicity has evolved at the height where previously a nematic phase was observed predominantly. This means that particles remain highly mobile so that in time continuing rearrangement of the particles occurs, causing a stronger fractionation and the growth of a pure smectic phase. The smectic phase might act as a kind of template for further growing this phase while retaining the same periodicity.

For this SAXS analysis a capillary with an initial volume fraction of $16 \%$ was used which might not seem justified to be compared with the capillary used for the TEM analysis (initial volume fraction of $8 \%$ ). Initially different liquid crystalline phases are observed, but in time similar behavior is

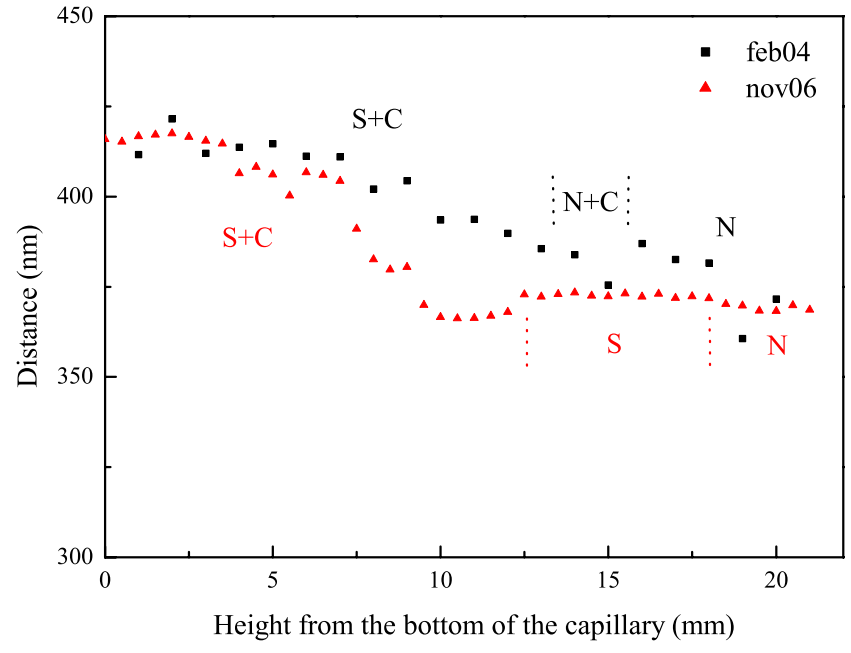

FIG. 6. (Color online) Small angle peak distances of the g55 system. 


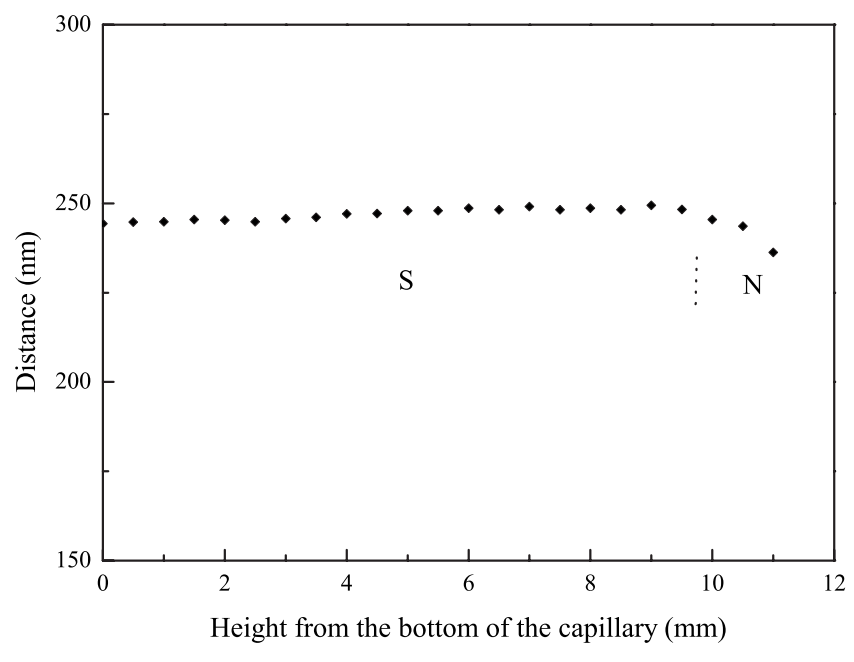

FIG. 7. Small angle peak distances of the g17 system.

observed. In both capillaries a smectic phase appears at a height where a nematic phase was observed before.

For the columnar distances in November 2006, there is also a small difference in the distances but there is less difference than for the smectic phase. The larger angle columnar peaks show a $4 \%$ increase in the distance going down in the capillary and the smaller angle peaks $8 \%$, compared to $13 \%$ for the smectic phase. An explanation might be that the double layer of the particles is more important for the width and the thickness than for the length. Therefore, less influence of the differences in width and thickness of the particles is expected on the distances between the columns.

Small angle distances were also measured in November 2006 for the g17 system (Fig. 7). A more or less constant distance is found in agreement with the constant particle length measured with TEM. The slight increase in distance going up in the sample might be counterintuitive because slightly smaller particles are expected there. This is probably caused by the higher osmotic pressure lower in the sample, thereby pushing the particles closer together, resulting in a smaller smectic periodicity.

\section{CONCLUSIONS}

Polydisperse $(35 \%$ and $55 \%$ ) dispersions of goethite form isotropic, nematic, smectic $\mathrm{A}$, and columnar phases. Because of the shape of our particles a biaxial nematic (or smectic) phase might be a possibility, but no conclusive evidence has been found for that. The existence of biaxial nematic phases in thermotropic liquid crystals has been a subject of heated debate ${ }^{40,41}$ and has been unambiguously demonstrated only recently for boomerang-shaped molecules. ${ }^{42,43}$ In our case, simulations ${ }^{44}$ of the phase diagram of the hard biaxial ellipsoid fluid (for which all three particle dimensions are different) are more relevant since the phase behavior of mineral liquid crystals is dominated by repulsions. These simulations indicate that only a narrow region of particle biaxiality, close to the point $L / W \approx W / T$ when ellipsoids are neither prolate nor oblate, leads to an actual biaxial nematic phase. Our particles do not fulfill this condition which might explain why no biaxial nematic phase was found. However, the fact that the columnar phases in these systems exhibit a distorted-hexagonal structure clearly indicates that the two smallest particle dimensions are not equivalent and particle biaxiality does play a role at higher concentrations.

The occurrence of a smectic phase is surprising because the terminal polydispersity to form a smectic phase was found to be $18 \%$ in simulations of hard spherocylinders. It was found by analysis of SAXS data and TEM measurements that sedimentation and fractionation play an important role. Regions within the sample were found with a length polydispersity almost twice as low as for the parent sample where the smectic phase was observed. Not only the average length increases going down the sample but also the aspect ratio. Furthermore, the width polydispersity does not hinder the formation of a columnar phase presumably because of the double layers and unequal width and thickness of the particles.

It is remarkable that over a few years large changes occur in the samples. Fractionation gets more pronounced in time and a pure smectic phase evolves. Brownian rearrangement of particles happens for a long time which means that particles remain highly mobile due to the double layer surrounding the particles.

The development of a pure smectic phase with a constant periodicity over about $5 \mathrm{~mm}$ of the sample is also remarkable. It might be possible that the smectic phase itself acts as a template for the new layers that form on top of it. But the smectic phase is mostly oriented with the particles oriented along the capillary and whole new layers can form on top of the old one. Therefore, at first sight there is no clear reason why the periodicity should remain constant over such a large distance.

In samples with a low polydispersity $(17 \%)$ also isotropic, nematic, and smectic A phases were found, but never a columnar phase. A columnar phase can accommodate particles with different lengths and therefore it is thought to act as a "waste disposal" for particles that do not fit into the smectic layers. This indicates that the smectic phase is the most stable phase and a columnar phase is only formed if the polydispersity does not allow the smectic phase to form.

A recently published new method for the synthesis of goethite nanotubes ${ }^{45}$ gives more monodisperse particles with tunable dimensions. This probably gives a nice model system for a further study of the phase behavior of monodisperse goethite.

\section{ACKNOWLEDGMENTS}

This work is part of the research programme SFB TR6 of the "Stichting voor Fundamenteel Onderzoek der Materie (FOM)," which is financially supported by the "Nederlandse Organisatie voor Wetenschappelijk Onderzoek (NWO)" and "Deutsche Forschungsgemeinschaft (DFG)." The work of D.M.E.T.-W. was performed as part of a NWO-CW TOP project. The staff of the BM26 DUBBLE beamline at ESRF is thanked for their support.

\footnotetext{
${ }^{1}$ H. Zocher, Z. Anorg. Allg. Chem. 147, 91 (1925).

${ }^{2}$ A. S. Sonin, J. Mater. Chem. 8, 2557 (1998).

${ }^{3}$ P. Davidson and J. C. P. Gabriel, Curr. Opin. Colloid Interface Sci. 9, 377
} 
(2005).

${ }^{4}$ J. C. P. Gabriel and P. Davidson, Adv. Mater. (Weinheim, Ger.) 12, 9 (2000)

${ }^{5}$ Q. Majorana, Rend. Accad. Naz. Lincei 11, 374 (1902).

${ }^{6}$ A. Cotton and H. Mouton, Ann. Chim. Phys. 11, 145 (1907).

${ }^{7}$ B. J. Lemaire, P. Davidson, J. Ferré, J. P. Jamet, P. Panine, I. Dozov, and J. P. Jolivet, Phys. Rev. Lett. 88, 125507 (2002).

${ }^{8}$ B. J. Lemaire, P. Davidson, J. Ferré, J. P. Jamet, D. Petermann, P. Panine, I. Dozov, and J. P. Jolivet, Eur. Phys. J. E 13, 291 (2004).

${ }^{9}$ B. J. Lemaire, P. Davidson, D. Petermann, P. Panine, I. Dozov, D. Stoenescu, and J. P. Jolivet, Eur. Phys. J. E 13, 309 (2004).

${ }^{10}$ B. J. Lemaire, P. Davidson, P. Panine, and J. P. Jolivet, Phys. Rev. Lett. 93, 267801 (2004).

${ }^{11}$ B. J. Lemaire, P. Davidson, J. Ferré, J. P. Jamet, D. Petermann, P. Panine, I. Dozov, D. Stoenescu, and J. P. Jolivet, Faraday Discuss. 128, 271 (2005)

${ }^{12}$ Z. Dogic and S. Fraden, Phys. Rev. Lett. 78, 2417 (1997).

${ }^{13}$ Z. Dogic and S. Fraden, Curr. Opin. Colloid Interface Sci. 11, 47 (2006).

${ }^{14}$ M. P. Lettinga, K. Kang, P. Holmqvist, A. Imhof, D. Derks, and J. K. G. Dhont, Phys. Rev. E 73, 011412 (2006).

${ }^{15}$ M. P. Lettinga and E. Grelet, Phys. Rev. Lett. 99, 197802 (2007).

${ }^{16}$ P. G. Bolhuis and D. A. Kofke, Phys. Rev. E 54, 634 (1996).

${ }^{17}$ P. Bartlett and P. B. Warren, Phys. Rev. Lett. 82, 1979 (1999).

${ }^{18}$ M. A. Bates and D. Frenkel, J. Chem. Phys. 109, 6193 (1998).

${ }^{19}$ L. Onsager, Ann. N.Y. Acad. Sci. 51, 627 (1949).

${ }^{20}$ H. N. W. Lekkerkerker, P. Coulon, R. Van Der Haegen, and R. Deblieck, J. Chem. Phys. 80, 3427 (1984).

${ }^{21}$ T. Odijk and H. N. W. Lekkerkerker, J. Phys. Chem. 89, 2090 (1985).

${ }^{22}$ A. Speranza and P. Sollich, J. Chem. Phys. 117, 5421 (2002).

${ }^{23}$ A. Speranza and P. Sollich, J. Chem. Phys. 118, 5213 (2003).

${ }^{24}$ A. Speranza and P. Sollich, Phys. Rev. E 67, 061702 (2003).

${ }^{25}$ H. H. Wensink and G. J. Vroege, J. Chem. Phys. 119, 6868 (2003).

${ }^{26}$ M. Fasolo and P. Sollich, Phys. Rev. Lett. 91, 068301 (2003).

${ }^{27}$ R. M. L. Evans, D. J. Fairhurst, and W. C. K. Poon, Phys. Rev. Lett. 81,
1326 (1998)

${ }^{28}$ F. M. van der Kooij, M. Vogel, and H. N. W. Lekkerkerker, Phys. Rev. E 62, 5397 (2000).

${ }^{29}$ H. H. Wensink and H. N. W. Lekkerkerker, Europhys. Lett. 66, 125 (2004).

${ }^{30}$ L. Bellier-Castella and H. Xu, J. Phys.: Condens. Matter 15, 5417 (2003).

${ }^{31}$ G. J. Vroege, D. M. E. Thies-Weesie, A. V. Petukhov, B. J. Lemaire, and P. Davidson, Adv. Mater. (Weinheim, Ger.) 18, 2565 (2006).

${ }^{32}$ D. M. E. Thies-Weesie, J. P. de Hoog, M. H. Hernandez Mendiola, A. V. Petukhov, and G. J. Vroege, Chem. Mater. 19, 5538 (2007).

${ }^{33}$ S. Krehula, S. Popović, and S. Musić, Mater. Lett. 54, 108 (2002).

${ }^{34}$ A. V. Petukhov, J. H. H. Thijssen, D. C. 't Hart, A. Imhof, A. van Blaaderen, I. P. Dolbnya, A. Snigirev, A. Moussaid, and I. Snigireva, J. Appl. Crystallogr. 39, 137 (2006).

${ }^{35}$ J. S. V. Duijneveldt and M. P. Allen, Mol. Phys. 90, 243 (1997).

${ }^{36}$ A. Stroobants, H. N. W. Lekkerkerker, and D. Frenkel, Phys. Rev. A 36, 2929 (1987).

${ }^{37}$ R. van Roij, P. Bolhuis, B. Mulder, and D. Frenkel, Phys. Rev. E 52, R1277 (1995)

${ }^{38}$ X. Wen, R. B. Meyer, and D. L. D. Caspar, Phys. Rev. Lett. 63, 2760 (1989).

${ }^{39}$ Z. X. Zhang and J. S. van Duijneveldt, J. Chem. Phys. 124, 154910 (2006).

${ }^{40}$ G. R. Luckhurst, Thin Solid Films 393, 40 (2001).

${ }^{41}$ G. R. Luckhurst, Nature (London) 430, 413 (2004).

${ }^{42}$ L. A. Madsen, T. J. Dingemans, M. Nakata, and E. T. Samulski, Phys. Rev. Lett. 92, 145505 (2004)

${ }^{43}$ B. R. Acharya, A. Primak, and S. Kumar, Phys. Rev. Lett. 92, 145506 (2004).

${ }^{44}$ P. J. Camp and M. P. Allen, J. Chem. Phys. 106, 6681 (1997).

${ }^{45}$ T. Yu, J. Park, J. Moon, K. An, Y. Piao, and T. Hyeon, J. Am. Chem. Soc. 129, 14558 (2007). 